\title{
Entstehungsmuster, Formen und Konstruktionen kulturellen Erinnerns/Vergessens bei Serben und Montenegrinern
}

\author{
HANS-MiCHAEL MiEDLIG (Göttingen)
}

Max Frisch hat einmal formuliert, ,Vergangenheit“ sei ,,eine Erfindung, die nicht zugibt, eine Erfindung zu sein, ein Entwurf rückwärts“. ${ }^{1}$ Der dahinterstehende Gedanke zielt auf das, was im Titel dieses Aufsatzes bereits anklingt und in der jüngeren und jüngsten kultursemiotischen Gedächtnisforschung sattsam diskutiert wurde bzw. wird: Jedes Individuum und jede soziale, ethnische bzw. nationale Großgruppe schaffen sich ihre jeweilige Vergangenheit aus einer bestimmten Gegenwart heraus, was nur über eine Rückbesinnung oder Erinnerung geschehen kann. So erscheint eben gerade auch die kollektiv wirksame kulturelle Erinnerung und ihre unabdingbare Voraussetzung, das Verges$\operatorname{sen}^{2}$, als unter den jeweiligen sozialen und politischen Bedingungen einer Zeit geschaffenes Produkt. ${ }^{3}$ Im Folgenden soll überblicksartig in einem vom Spätmittelalter bis heute gespannten Bogen verdeutlicht werden, wie und zu welchem Zweck kulturelle Erinnerung bei bzw. von den Serben und Montenegrinern ,gemacht“ wurde und wird. Im Einklang mit Ludwig Steindorff wird es nicht darum gehen, das kulturelle Gedächtnis der vormodernen Zeit im Sinne einer langlebigen und vieldimensionalen Gedächtniswelt, als „Kultur der Memoria“, wie Otto Gerhard Oexle es nennt ${ }^{4}$, ihren Nachfolgern, d.h. den

1 Zitat nach Fromm / Haase / Schlottke 2003, S. 7.

2 Zur grundsätzlichen Problematisierung von kulturellem Vergessen Butzer / Günter 2004. A. Erll hat das Verhältnis von Erinnern und Vergessen, besonders in seiner kollektiven Form, auf der Grundlage der einschlägigen Forschung treffend folgendermaBen paraphrasiert: „Erinnern und Vergessen sind zwei Seiten - oder verschiedene Prozesse - desselben Phänomens: des Gedächtnisses. (Soziales) Vergessen ist Voraussetzung für (kulturelle) Erinnerung. Denn total recall, die lückenlose Erinnerung an jedes einzelne Ereignis der Vergangenheit, käme für das Individuum ebenso wie für die Gruppe oder die Gesellschaft dem totalen Vergessen gleich“. Erll 2005, S. 7.

3 Dazu Eckart Olshausen: „Bezüge auf die Vergangenheit, auf die Gegenwart und auf die Zukunft sind in den verschiedenen Gesellschaften zu verschiedenen Zeiten ganz unterschiedlich häufig zur Hand, dienen ganz unterschiedlichen Zwecken und können, genau betrachtet, die jeweilige Gesellschaft ganz wesentlich charakterisieren“. Olshausen 2003, S. 159.

4 Vgl. Oexle 1995, S. 9-78. 
weniger komplexen „Gedächtnisorten“ der Moderne, schroff gegenüberzustellen. ${ }^{5}$ Gerade das Beispiel der Serben und Montenegriner zeigt vor dem Hintergrund der jüngsten Kriege im ehemaligen Jugoslawien, dass eine solche Trennung der ,vormodernen“ von den „modernen“ kollektiven Gedächtnisstrukturen hier nur sehr bedingt Sinn macht. Es werden nämlich im Verlauf der Jahrhunderte sehr zählebige Gedächtnisse sichtbar, die eine ferne, religiös konnotierte Vergangenheit schließlich auch mit dem nationalen Gedanken strukturell verbinden. Als gutes Beispiel kann die Kosovo-Legende dienen, von der noch die Rede sein wird.

Im mittelalterlichen serbischen Feudalstaat entstanden außer sakralen architektonischen Bauwerken und Artefakten der religiösen Sachkultur eine Reihe von hochkulturellen Gedächtnisorten. Dazu gehört vor allem die kirchliche bzw. kirchlich vermittelte Literatur, einschließlich der Heiligenlegenden, religiösen Poesie sowie der Lebensbeschreibungen mittelalterlicher Herrscher. Von diesen schriftlichen Manifestationen des kulturellen Gedächtnisses fanden die meisten noch bis zum Ende des 19. Jahrhunderts, ja darüber hinaus, nur indirekt Eingang in die große bäuerliche Bevölkerungsmehrheit. Dies ist in einer dominant illiteraten Gesellschaft nicht verwunderlich. Dennoch konnten sich einige dieser Artefakte im gegebenen Gedächtnismilieu durch orale Tradierung etablieren, z.B. die Ende des 13. Jahrhunderts entstandene Lebensbeschreibung des Heiligen Sava durch den Mönch Teodosije, oder das im Auftrag des serbischen Patriarchen Nikon 1431 verfasste bedeutsame biographische Werk aus der Feder Konstantins des Philosophen: Das Leben Stefan Lazarevićs, des Sohnes des serbischen Fürsten Lazar, der 1389 auf dem Amselfeld umkam. ${ }^{6}$ Somit sind wir bei zwei wichtigen erinnerungsstiftenden Motivelementen bei Serben und Montenegrinern: Die Kolportierung des Sava-Bildes durch Teodosije war einer der ersten zentralen „Bausteine“ für die Entwicklung, Erhaltung und Bewahrung religiöser und ethnischer Identität, die Erinnerung Konstantins an die Kosovo-Schlacht gegen die Osmanen und den „Heldentod" Fürst Lazars wob am Bild des im kollektiven Bewusstsein allmählich entstehenden Kosovo-Mythos. ${ }^{7}$ Der Lazar-Kult wurde von der orthodoxen Kir-

5 Steindorff will in bewusster Abgrenzung von Pierre Nora ausdrücklich keine so scharfe Grenze gezogen sehen. Dennoch gebe es natürlich solche grundsätzlichen Unterschiede zwischen den beiden „Zeitaltern des Gedächtnisses“, denn es werde ,[...] mit der Säkularisierung und Pluralisierung der Gesellschaft immer schwieriger, die aus einer Vielzahl von Orten zusammengesetzte Gedächtniswelt oder Gedächtniskultur zusammenzuhalten, sie löst sich allmählich in vereinzelte Orte auf und wird immer labiler. An die Stelle der jahrhundertelang funktionierenden, stabilen, religiös begründeten Gedächtnisorte sind [nunmehr in der „Moderne“ - Erg. v. H.-M. Miedlig] die in ihrer Beständigkeit viel flüchtigeren Gedächtnisorte der Nation getreten“. Vgl. Steindorff 2003, S. 159.

6 Zur Lebensbeschreibung des heiligen Sava vgl. Stefanović / Stanisavljević 1968, S. 116, 117. Zum zweitgenannten Werk Braun 1956.

7 Vgl. zu Letzterem Emmert 1990, S. 61-78. 
che gezielt gepflegt, zumal sie Fürst Lazar nachträglich als Heiligen kanonisiert hatte. ${ }^{8}$ Die Funktion der Stiftung bzw. Erhaltung der serbischen ethnischen Gemeinschaft durch Erinnerungswahrung über die Jahrhunderte der osmanischen Fremdherrschaft seitens der serbisch-orthodoxen Kirche ist denn auch in der Forschung zu Recht betont worden. ${ }^{9}$

Von ganz besonderer Wichtigkeit für die Programmierung des serbisch-montenegrinischen kulturellen Gedächtnisses waren allerdings bestimmte Formen und Institutionen der oralen teils bäuerlichen teils pastoralen patriarchalischen Kultur. Sie wurden im Zuge der großen Migrationen als mittel- oder unmittelbare Folge der osmanischen Eroberungszüge aus dem Kosovo und dem heutigen Makedonien nach Norden und Nordwesten, u.a. auch nach Montenegro, nach Nordbosnien, Südkroatien, die serbische Šumadija sowie die Vojvodina transportiert. Zum ersten sei in diesem Zusammenhang an den berühmten Serbenzug unter Führung des Patriarchen Arsenije III. 1690 nach dem Rückzug der österreichischen Heere im großen Türkenkrieg 1683-1699 erinnert. Die Mönche des Klosters Ravanica in Südserbien nahmen damals die dort aufbewahrten Gebeine des Fürsten Lazar als Erinnerungsort ersten Ranges mit bei ihrem Exodus nach Südungarn. ${ }^{10}$ Zum zweiten muss an dieser Stelle auch auf die einschlägigen Folgen des Zustroms montenegrinischer und serbisch-bosnischer Bevölkerungsteile in das nun autonome und deshalb relativ sichere Zentralserbien im Verlauf der ersten Hälfte des 19. Jahrhunderts verwiesen werden. ${ }^{11}$

Auch hier geht es um den Transfer historisch-kultureller Erinnerung und damit um deren (Ver-)Stärkung im Bewusstsein der serbischen Altsiedler. Welche wichtigen oralen Kulturformen der Memoria wurden hier transportiert? Einerseits handelte es sich um regionale und überregionale Gedächtnisse, die sich in bestimmten religiösen Rechts-, Fest- und Alltagspraktiken sowie volksliterarischen Artefakten, etwa Volkserzählungen, lyrischen und vor allem epischen Liedern ${ }^{12}$, niederschlugen. Um Letztere soll es im Folgenden gehen, vermittelte sich in ihnen kollektive Erinnerung durch regelmäßige Wiederholung doch in geradezu paradigmatischer Weise. So wurden bewusst wie unbewusst Konstruktionen gebaut, die auf die ethnos- bzw. gemeinschaftsbildenden

8 Zirojević 1999, S. 45.

9 Vgl. Richter 1999, S. 384, 385; Sundhaussen 1993, S. 18.

10 Nitsche 2003, S. 147.

11 Hierzu Miedlig 1991, S. 169.

12 Die große Bedeutung der epischen Tradierung in ihrer Auswirkung auf das kollektive Bewusstsein der Bevölkerung lässt sich z.B. an den Beobachtungen des Frankfurter Sprachwissenschaftlers P.A. Possart ablesen: „Zur Volksmusik gehört [...] die serbische Geige, Gusle genannt, zu deren Begleitung man Heldenlieder singt. In jedem Dorfe gibt es einen Dudelsackpfeifer, Geiger aber, besonders in den bergigen Gegenden gegen Bosnien und Herzegowina zu, giebt es beinahe in jedem Hause. Fast alle Geschäfte verrichten die Serbier singend. Die Hirten singen auf den Bergen und in den Wäldern“. Vgl. Possart 1837, S. 74. 
Bezüge zum gemeinsamen orthodoxen Glauben und zu vergangenen historischen Ereignissen rekurrierten. Erinnerung vollzog sich dabei in den Kategorien der patriarchalisch-heldischen, moralisch absolut verbindlichen Denk- und Verhaltensschemata der dem Gewohnheitsrecht verpflichteten segmentären Stammesverbände im Westen (s. Montenegro) bzw. der fratristischen, später patrilinearen dörflichen Familiengemeinschaften im Osten (s. serbische Sumadija). Von diesen Volksepen, die die Forschung in neun „Zyklen“ systematisiert hat, gingen selbstverständlich nicht alle mit ihrer Botschaft dauerhaft in das kollektive Gedächtnis der Serben und Montenegriner ein. „Mentale Eintragungen“, wie es Rudolf Jaworski nennt ${ }^{13}$, können sich nur dann in diesem Gedächtnis verankern, wenn sie funktional, d.h. auf Identitätsstiftung bzw. -bewahrung angelegt sind. Im Folgenden sollen - um nur das Wichtigste zu nennen - zunächst die epischen Überlieferungen über den Heiligen Sava und die Nemanjiden-Herrscher, zweitens über die Folgen osmanischer Eroberung und die Auseinandersetzung mit den neuen Gewalten betrachtet werden. Im Zentrum steht hier ein auch für die Montenegriner genauso bedeutsamer Gedächtnisort, nämlich die serbischen Aufstände 1804-1815. ${ }^{14}$ Drittens ist die aufgrund ihrer dominanten Stellung im kulturellen Gedächtnis zentral wichtige und deshalb besonders zu hinterfragende Kosovo-Legende anzuführen.

Schon Maximilian Braun hat erkannt, dass das serbische Heldenlied grundsätzlich nichts erfindet, „es berichtet“, so Braun, „über tatsächliches Geschehen“, allerdings in epischer Umgestaltung, wodurch letztlich eine "gesteigerte Wirklichkeit" vermittelt werde. ${ }^{15}$ Dem epischen Sänger geht es darum, das dem Publikum Mitgeteilte in agonalen Dichotomien darzustellen. Kampf ist also nie Schlachtgetümmel in nüchterner Erzählung eines mehr oder weniger komplexen Ereignisablaufs, sondern immer eine heldische Auseinandersetzung zwischen zwei Kontrahenten oder zwei genau eingegrenzten Gruppen. In den Epen über Kirche und Staat im Mittelalter tritt der heilige Sava deshalb in heldischer Manier als Kämpfer gegen die Nichtchristen auf. Das Ziel des Sängers ist es, stolz die Erinnerung an den Kirchengründer Sava sowie das alte

13 Jaworski 2003, S. 12.

14 Die Montenegriner betreffend vgl. in diesem Zusammenhang Dašić 1983, insbes. S. 590, 594. Zur bewusstseinsbildenden Breitenwirkung der heroisierenden Abbildung des Aufstandsführers Karađorđe im „Bergkranz“ des Dichterfürsten Petar Petrović II. Njegoš sowie den serbischen bzw. montenegrinischen Heldenliedern, vorgetragen von dem herausragenden Volkssänger Filip Višnjić s. Kilibarda 1983, S. 789, 792. Weiteres hierzu S. 235 dieses Aufsatzes.

15 Vgl. Braun 1961, S. 28, 29, 40. Über die heldische Gestaltung und Bearbeitung der historischen Wirklichkeit in der serbischen Volksepik ,unter ständiger Kontrolle durch die epische serbische Gemeinschaft" vgl. die konzise Charakteristik bei Durić 1996, S. 4, 5. Ungeachtet dieser Transformation der Wirklichkeit orientierten sich die Inhalte der Epen, z.B. Schlachtbeschreibungen, vor dem Hintergrund des Gesagten allerdings zuweilen durchaus an den konkreten historischen Fakten. Vgl. Ljušić 1994, S. 380-382. 
serbische Reich und seine Größe wach zu halten. Auch wird mahnend des selbstverschuldeten Streits zwischen den einzelnen serbischen Teilfürsten nach Stefan Dušans Tod und des dadurch bewirkten, als tragisch-schicksalshaft dargestellten Untergangs noch vor der osmanischen Invasion gedacht. ${ }^{16}$ Hier ist eines der „Bauelemente“ für jenen über die Jahrhunderte entwickelten kollektiven Bewusstseinszustand der Serben zu suchen, den Gabriella Schubert treffend mit dem Ausdruck ,tragisches Geschichtsbewusstsein“ beschrieben hat. ${ }^{17}$

Belege darüber, wie sehr sich der glorifizierte mittelalterliche serbische Großstaat als Fixpunkt der kulturellen Erinnerung verankert hat, finden sich im politischen Denken Arsenijes III. ${ }^{18}$ genauso wie auch in der Ideenwelt des serbischen Aufstandsführers Karađorđe Petrović. Zusammen mit lokalen Sippenältesten rief Letzterer in einem an den herzegowinischen Archimandriten von Piva gerichteten Brief vom August 1804 zum gemeinsamen Kampf um die Wiedergewinnung des alten serbischen Landes auf. Zwei Jahre später sprach er in einem ähnlichen Aufruf an die Montenegriner vom ,verlorenen serbischen Staat", der wiedererkämpft werden müsse. ${ }^{19}$ Typischerweise finden sich solche bewusst erinnernden Rückbezüge auf das serbische mittelalterliche Staatswesen besonders im entwickelten Nationalismus der zweiten Hälfte des 19. Jahrhunderts. Um ein Beispiel zu nennen: Vor, in und nach den Kriegen des Osmanischen Reichs mit Serbien, Montenegro sowie in der Folge auch mit Russland 1876-78 und der Entstehung zweier unabhängiger serbischer Staaten auf dem Berliner Kongress 1878 brach zwischen diesen ein vergangenheitsorientierter geostrategischer Konkurrenzkampf aus. Es ging darum, wer befugt sei, angesichts der offensichtlichen Schwäche des osmanischen Reichs Anspruch auf die alten serbischen Länder und Landschaften des Reichs Stefan Dušans, gewissermaßen als dessen legitimer Rechtsnachfolger, zu erheben. Dazu gehörte, diese Gebiete dem eigenen Staatswesen ggf. einverleiben zu können, kurzum, es ging um den Ort und die Dynastie eines neuen „serbischen Piemonts“. ${ }^{20}$ In der bereits nationalistisch manipulierten, selektiv operierenden öffentlichen Erinnerung blieb dabei ausgeblendet (und wurde vergessen!), dass das alte serbische Zartum keineswegs ein unitarer serbischer Nationalstaat, vielmehr multiethnisch strukturiert war, vom modernen „Prinzip Nation“ noch weit entfernt.

Der Sava-Mythos ging als Kult nicht nur in das amtskirchliche, sondern auch in das breite Bevölkerungsschichten erfassende kollektive Gedächtnis ein,

16 Stefanović / Stanisavljević 1968, S. 35-39.

17 Schubert 1996, S. 157.

18 Vgl. Veselinović 1976, S. 50.

19 Vgl. Petrović 1976, S. 58.

20 Vgl. Miedlig 2006, S. 161, 162. Implizit wurden hier die politischen Zielvorstellungen, die der ehemalige serbische Innenminister Ilija Garašanin in seinem berühmten Geheimprogramm von 1844 für Serbien formuliert hatte, erinnert. Es ging um die Lösung der „serbischen Frage“ auf dem Wege der Wiederherstellung des alten serbischen mittelalterlichen Großreichs, s. hierzu Sundhaussen 2001, S. 28. 
besonders jüngst in die nationalistische serbische Erweckungsbewegung seit Mitte der 80er Jahre des 20. Jahrhunderts. ${ }^{21}$ Hier spielt die neu geschaffene patriotisch-politische Volkspoesie, mit dem Heiligen Sava als Führer- und Leitfigur, eine herausragende Rolle. ${ }^{22}$ In den jugoslawischen Zerfallskriegen sollte der Name des Kirchengründers zum folkloristischen Etikett serbischer Kriegshelden, verewigt in Trivialromanen und Comic Strips, werden, wobei der Publizist Predrag Milošević den heiligen Sava zum ersten heiligen Krieger und Lehrer der Kampftechniken unter den Serben stilisierte. ${ }^{23}$

In den Liedern über die Auseinandersetzung mit der osmanischen Gewalt kommt das an andere Generationen weiterzugebende heldische Prinzip u.a. dadurch zum Ausdruck, dass die Leitlinie des Denkens und Handelns aller Kämpfer heißt, lieber stehend wie ein Mann zu sterben, als sich vor dem Feind $\mathrm{zu}$ beugen und unwürdig in dessen Gewahrsam weiterzuleben. So etwa im Poem Tod des Vojevoden Prijezda. ${ }^{24}$ In den serbischen Freiheitskämpfen 1804-1815 war dieses heldische Prinzip fest verinnerlichte, d.h. stets neu erinnerte und befolgte Praxis. Die besondere Nachhaltigkeit dieser Denk- und Handlungsdisposition und deren tiefe Speicherung im kollektiven Gedächtnis zeigte sich im Übrigen auch nach der serbischen Niederlage im Ersten Weltkrieg, als Ministerpräsident Nikola Pašić den Satz prägte: „Besser wir sterben alle als freie Menschen, als dass wir wie Sklaven leben ". ${ }^{25}$

Das Gestaltungsprinzip des heldisch-agonalen Zweikampfs wurde in den Erzählungen über den serbischen Aufstand konsequent beachtet. Im Epos über die Schlacht bei dem Dorf Mišar am 13. August 1806, in der die serbischen Einheiten unter Karađorđe einen ersten glänzenden Sieg über die bosnischosmanischen Elitetruppen errangen, erscheint dieser Zweikampf als Auseinandersetzung zwischen den jeweils besten Helden auf beiden Seiten. Für den Zuhörer hatte diese Erzählung zum Spiel der Gusle ${ }^{26}$ ethisch-paradigmatischen

21 Sundhaussen 2001, S. 20, 33. In einem wichtigen Politika-Artikel der Umbruchszeit wurde die „Sveta-Sava-Ideologie, als essentieller Inhalt der serbischen Geistigkeit“, als „ausgesprochene Lebensnotwendigkeit“ umschrieben. „Die serbische historische Erinnerung" manifestiere sich gerade in der ,größten christlichen Kathedrale des Balkans“ auf dem Vračar, wo einst Sinan-Paşa den Körper des heiligen Sava verbrannt habe. „Auf diese Weise, durch die Persönlichkeit Savas“ erhärte sich „die Identität und Integrität der serbischen Nation“. Vgl. Živanović 1991, S. 13.

22 Hierzu Näheres bei Čolović 1994, S. 90, 91.

23 Ebd., S. 127, 130, 131.

24 Stefanović / Stanisavljević 1968, S. 52.

25 Vgl. Zirojević 1999, S. 51.

26 Es ist wichtig, hervorzuheben, dass dieses Streichinstrument (einseitige Kniegeige) nie ausschließliches „Eigentum“ der serbischen Volkskultur war. Auch bei den Kroaten und bosnischen Muslimen waren epische Gesänge zum Spiel der Gusle beliebt. Trotz vorangegangener Versuche in entsprechender Richtung wurde die Kniegeige im Wesentlichen erst in den jüngsten jugoslawischen Zerfallskriegen 1990-1995 gezielt zum 
Charakter. Die Übermittlung der Botschaft eines solchen Sieges drückte sich tief in das kollektive Gedächtnis ein. ${ }^{27}$ Dies gilt insbesondere, wenn sogar noch ein Zeuge der Ereignisse, wie es der berühmte Sänger Filip Višnjić war, als Erzähler auftrat. Višnjić war zudem für seine Zuhörer selbst ein Denkmal und Erinnerungsort sui generis. Indirekt trug er dazu bei, die kollektive Erinnerung an die serbischen Freiheitskämpfe auch in Montenegro wach zu halten: Der Dichterfürst Petar Petrović II. Njegoš kleidete seine Verehrung für Karađorđe in eine seinem „Bergkranz“ vorgeschaltete romantische Ode an den unter Nutzung der Epen Višnjićs glorifiziert-überhöht dargestellten Aufstands-führer. ${ }^{28}$

Die kollektive Erinnerung an die großen serbischen Aufstände vollzog sich zunächst in Gruppengedächtnissen der traditionalen Gesellschaft. Es handelte sich um lokale Großfamilien- und Sippengedächtnisse, in denen sich die Erinnerungen an örtliche angesehene Hausvorsteher speicherten, die an den Kämpfen in den jeweiligen Gegenden teilgenommen hatten. ${ }^{29}$ Neben diesem illiteraten patriarchalischen bäuerlichen Milieu mit seiner ausschließlich oralen Kultur entstanden noch im 19. Jahrhundert allmählich neue lieux de memoire in den vorerst noch dünnen städtischen Bevölkerungsschichten. Da die sich bildende bürgerliche Intelligenz ihre Herkunft überwiegend vom Lande ableite$\mathrm{te}^{30}$, war auch in diesem Milieu die Erinnerung an die oralen epischen Traditionen des Dorfes präsent. Daher konnten die ersten Theateraufführungen zu den großen Ereignissen der serbischen Befreiungskriege als wichtige Träger kultureller Erinnerung funktionieren. $\mathrm{Zu}$ nennen sind hier die noch unter Fürst Miloš Obrenović, dem Führer des zweiten Aufstands 1815, aufgeführten Dramen Die Wiederherstellung Serbiens und Der Kampf bei Čačak. 1847/1848 wurden die Theateraufführungen zur genannten Thematik fortgesetzt, so mit Karađordes Apotheose von Đorđe Maletić, mit Sterija-Popovićs Dramen Die Feier Serbiens sowie Der Traum bzw. Säbel des Königssohns Marko. Im letztgenannten Stück findet sich jener bedeutsame Aufruf: „Steh auf, steh auf, Serbe, auf zu den Waffen!" Diese Aufführungen mit ihren im Stil der Zeit romantisiert verklärten Themen aus den Befreiungskämpfen hatten beachtlichen $\mathrm{Zu}$ lauf beim Publikum. Sie stellten wie andere Dramen, etwa Đura Jakšićs Stanoje Glavaš, auch weiterhin beliebte Erinnerungsorte dar. ${ }^{31}$ Es ist wichtig, zu betonen, dass sich nicht erst Slobodan Milošević, sondern schon die serbischen Regierungen bzw. Fürstenhäuser seit der Zeit des aufkommenden nationalen

exklusiven serbischen Nationalinstrument erhoben und ideologisiert. Dazu Žanić 1998, S. 51-56.

27 Vgl. Braun 1961, S. 41-44.

28 Dazu Kilibarda 1983, S. 788-794; s. a. Anm. 14 dieses Aufsatzes.

29 Dazu Alimpić 1852, S. 3, 4.

30 In diesem Sinne für Belgrad bestätigend: Miljković 1977, S. 73. Vgl. hier auch die Lebenswege wichtiger Persönlichkeiten aus der serbischen Intelligenz, etwa diejenigen Jovan Skerlićs, Jevrem Grujićs und vieler anderer.

31 Nikolić 1983, S. 813-819, 826. 
Patriotismus in den 60er Jahren des 19. Jahrhunderts als politische Manipulateure und absichtsvolle Sponsoren kulturellen Erinnerns betätigten. So sollten am Tag der Inthronisierung Milan Obrenovićs 1872 zwar die alten oben genannten Stücke über den ersten Aufstand aufgeführt werden, auf höchsten Befehl wurden nun jedoch Karađorđe und Stanoje Glavaš durch Miloš Obrenović ersetzt. Es ging darum, ausschließlich der Dynastie Obrenović ein bleibendes Denkmal zu setzen. 1898, zum Geburtstag des Königs, erfolgte eine erneute öffentliche Zelebrierung Milošs als eines Obrenovićs im Kontext des zweiten Aufstands. Nach dem Dynastiewechsel 1903 standen nun wieder Karadorde und der erste Aufstand ganz oben auf der staatlichen Erinnerungsagenda. Letztere setzte sich bis zum Zweiten Weltkrieg fort. ${ }^{32}$ In der Zeit zwischen 1945 bis Anfang der 80er Jahre konnten dann trotz oder gerade wegen der denational ausgerichteten Staatsideologie in freiheitskämpferischer Deutung Stücke sowohl über den ersten wie über den zweiten serbischen Aufstand aufgeführt und wissenschaftliche Symposien zu den Jahrestagen der ,serbischen Revolution“" veranstaltet werden. Unter den dramatischen Inszenierungen der serbischen Befreiungskriege seien in diesem Zusammenhang besonders $\mathrm{J}$. Veselinovićs Hajduk Stanko, erneut Đ. Jakšićs Stanoje Glavaš sowie Ivan Studenis Vo žd (Führer) erwähnt. Diese Aufführungen hatten als Beschwörung der Widerstandskraft und Wiederauferstehung eines unterdrückten Volkes im Türkenkampf eine wichtige Funktion in der kollektiven historischen Erinnerung. Damit passten sie gut in die herrschende staatliche Integrationsideologie $^{33}$, in der der Volksbefreiungskampf im Zweiten Weltkrieg mit der für alle jugoslawischen Völker im Zweiten Jugoslawien verbindlichen volksrevolutionären Ethik eine entscheidende Rolle spielte. ${ }^{34}$

Die politische Einflussnahme auf die öffentliche Erinnerung, d.h. die Formierung des kollektiven Gedächtnisses durch Lancierung der Heldenbilder und -symbole aus den serbischen Befreiungskriegen, wurde bekanntermaßen jedoch erst mit dem Aufstieg Slobodan Miloševićs massen-mobilisatorisch perfektioniert. ${ }^{35}$ Mitte der 80er Jahre des 20. Jahrhunderts gerieten die Aufstände 1804-15 zum sinnstiftenden Moment in einer krisenhaften Gegenwart, sozusagen als ,agonales Selbstbild“36 aller Serben. Dieses hängte sich an die Konstruktion, Verbreitung und Rezeption der nun zu besprechenden KosovoLegende an. Sie gilt mit vollem Recht nicht nur als „Nationalmythos der Ser-

32 Ebd., S. 820-825.

33 Ebd., S. 826-828.

34 Zur offiziellen titoistischen Erinnerungsagenda mit ihren spezifischen ideologischen Werten, für deren Legitimität der 2. Weltkrieg ,ausschließlich“ als „Instrument“ in Anspruch genommen wurde, vgl. Hoepken 1999, S. 196-205.

35 Näheres dazu bei Popović 2003. Vgl. im Übrigen Durić 1996, S. 20.

36 Höpken 2001, S. 387. 
ben“, sondern auch als deren ,untrennbarer Bestandteil historischer Erinnerung über die Jahrhunderte hinweg". ${ }^{37}$

Die Überlieferung über die Amselfelder Ereignisse ist leider nur lückenhaft und die erhaltenen historischen Quellen über die Schlacht der serbischbosnisch-kroatisch-albanisch-walachisch-bulgarischen Verbände unter Führung des Fürsten Lazar Hrebeljanović gegen das Heer Sultan Murads I. am sog. St. Veitstag, dem 15./28. Juni 1389, erweisen sich als wenig aussagekräftig. Es ist noch nicht einmal sicher, ob der Sultan tatsächlich einen Sieg davontrug. Aus den Quellen, die unmittelbar nach der Schlacht entstanden, geht lediglich hervor, dass Murad nach dem Ende der Kämpfe, - wohl von einem serbischen Adligen $^{38}$ - noch auf dem Amselfeld ermordet, Fürst Lazar festgesetzt und hingerichtet wurde. Auch in einer ragusanischen Quelle vom Ende des 15. Jahrhunderts ist nach wie vor von einem auf beiden Seiten nicht entschiedenen Sieg die Rede. ${ }^{39}$

Die Lücken reizten dazu, gefüllt zu werden, denn es ging auch um eine bleibende Rechtfertigung der im serbischen kollektiven Gedächtnis als Katastrophe empfundenen Niederlage. Die viel wichtigere Zweite Amselfelder Schlacht vom 18./19. Oktober 1448 (Janos Hunyadi gegen Murad II.) blieb und bleibt demgegenüber vergessen. Als Grund könnte angenommen werden, dass sie ein für das kulturelle Gedächtnis nicht funktional ausdeutbares und deshalb auszusortierendes Konkurrenzereignis war und weiterhin ist.

Die so im Verlauf der Jahrhunderte zunehmend mythisch ausgestaltete Überlieferung der Amselfelder Erzählung speist sich aus zwei „Schichten“, einer religiösen und einer volksliterarischen. Diese mischten sich in charakteristischer Weise: Die Wirkung des hagiographischen Schrifttums der serbischorthodoxen Kirche zeigt sich im Poem Der Untergang des serbischen Zartums darin, dass Fürst Lazar vor dem Kampf der Heilige Elias in Gestalt eines grauen Falken erschien, mit einem Brief der Gottesmutter. In diesem wurde Lazar vor die Wahl gestellt, sich entweder durch Sturm auf das Türkenheer in Siegeshoffnung für das kurzlebige irdische oder aber bewusst für das ewigen Lohn verheißende himmlische Reich zu entscheiden. Letzteres bedeutete selbstverständlich den irdischen Tod. ${ }^{40}$ Der Fürst wählte das Zweite und traf damit - so

37 Zitate nach Richter 1999, S. 381.

38 Dass es sich hierbei um Miloš Obilić handelte, ist eine nachträglich erfundene Legende. Vgl. Zirojević 1999, S. 46. Die mangelnde historische Identifizierung Obilićs sowie auch das Zeugnis einer florentinischen Quelle vom 20. Oktober 1389 über die Ermordung Murads durch einen ,,jener zwölf Edlen“, die sich den Weg zum Zelt des Sultans mit dem Ziel von dessen Ermordung gebahnt hätten, verdeutlicht Sundhaussen 2001, S. 21, 26 bzw. 19.

39 Sundhaussen 2001, S. 18-22 mit den wichtigsten Quellenbelegen. Den quellenmäßig nicht sicher belegten Verlauf sowie auch das bis heute nicht eindeutig feststellbare Ergebnis der Schlacht betont ferner Šuber 2004, S. 349, 357.

40 Dass dieser durch das Schwert eines heidnischen Herrschers zu erleiden sein würde, war „für die Kanonisierung des Fürsten von besonderer Bedeutung“, denn dadurch 
die Erzählung - eine gottgefällige Entscheidung. Er trat in die Schlacht, die er, vorbestimmtermaßen, durch den Verrat seines Schwiegersohnes Vuk Branković verlor. So entwickelte sich in der Erinnerung breiter Volksmassen allmählich eine mythische Vorstellung von der Erfüllung des serbischen Schicksals, mit dem selbst gewählten Opfertod Lazars in einem serbischen Golgatha als Beginn „einer jahrhundertelangen Leidensgeschichte des serbischen Volkes“, wie es Peter Nitsche ausdrückt. Die Wirkung der amtskirchlichen Deutung zeigt sich auch darin, dass Lazar als Märtyrer und zweiter Christus erscheint, während der gleichfalls ganz unhistorische Verrat des Vuk Branković zum biblischen Judasverrat gerät. ${ }^{41}$

Die in der Forschung auch „mystisch-heroisch“ genannte volksliterarische „Schicht“ der epischen Überlieferung schuf mit dem angeblichen Sultansmörder Miloš Obilić eine den gesellschaftlichen Strukturen entsprechende Figur. Denn dessen Tat entsprach ganz der herrschenden Vorstellung in den serbischen und montenegrinischen Bauern- bzw. Hirtengesellschaften von einer absolut gerechten, ethisch einwandfreien heldisch-agonalen Racheaktion zugunsten der Gemeinschaft. ${ }^{42}$

Dass die volksliterarische Überlieferungsschicht durch den serbisch-orthodoxen Klerus theologisch umgeformt und überhöht wurde, belegt nicht zuletzt folgende Tatsache: Die in den Vukschen Volksliedsammlungen bewahrte ursprüngliche Fassung des Kosovo-Epos spricht noch von einem Brief Murads I. mit der Herausforderung an König Lazar, sich zum Kampf um das Kosovo, das nicht zwei Herren haben und „doppelte Steuer“ zahlen könne, zu stellen, es sei denn, dass Lazar sich vorher ergäbe. ${ }^{43}$

Die bis zum Beginn des 18. Jahrhunderts in der Volksepik sowie der Prosaüberlieferung ausmodellierte und im kulturellen Gedächtnis aufbewahrte Amselfelder Legende ${ }^{44}$ entwickelte sich bis zum Ende des 19. Jahrhunderts zum serbischen Nationalmythos. ${ }^{45}$ Er wuchs im Zusammenhang mit der Entstehung

wurde „der neue Leidende auf eine Stufe mit den frühchristlichen Märtyrern gestellt“. Zitiert nach: Knez Lazar, in: 100 najznamenitijih Srba. 1993, S. 51 bei Richter 1999, S. 385.

41 Vgl. Nitsche 2003, S. 142-144, Zitat S. 145; Zirojević 1999, S. 47.

42 Sundhaussen 2001, S. 21, unter Verweis auf die „Literaturarchäologie“ Miodrag Popovićs ferner S. 26.

43 Durić 1996, S. 8.

44 Die Legende in ihrer Endform stellt die durch unhistorische Elemente der montenegrinischen Überlieferung erweiterte Fassung des Amselfelder Mythos, wie er bis heute rezipiert und gepflegt wird, dar. Diese Fassung geht auf den Text einer von einem unbekannten Autor des 18. Jahrhunderts aus der Gegend um Kotor verfassten Heiligenvita des Fürsten Lazar, Miloš Obilić und der übrigen Adelsherren, die auf dem Amselfeld waren zurück. In der Forschung wird sie auch unter dem Titel Geschichte über die Amselfelder Schlacht behandelt. Vgl. Zirojević 1999, S. 47, 48.

45 Dennoch war dieser Mythos keineswegs immer der alleinige geistige nationale Besitz der Serben. Auch unter den Nachbarvölkern wurde er zumindest phasenweise, etwa in 
der antiosmanischen nationalen Emanzipations- bzw. Erweckungsbewegungen seit dem Beginn der beiden erwähnten serbischen Aufstände und unterlegte die sog. ,serbische Frage“. ${ }^{46}$ Die in der Legende übermittelten Botschaften wurden im kollektiven kulturellen Gedächtnis fest verankert, so dass die Wahl des „Himmlischen Reichs“, mit ehrenvollem Tod statt „Leben in Schande“ und damit der Verzicht auf das Kosovo als Beginn des erwähnten ,jahrhundertelangen Leidens" verinnerlicht wurde. Man vergaß, dass die osmanische Herrschaft im Gegenteil durchaus von Toleranz geprägt war. ${ }^{47}$ Auf dieses letztgenannte Faktum braucht angesichts der Eindeutigkeit der neueren westlichen Forschungsergebnisse hier nicht näher eingegangen zu werden. So zeigt sich erneut der selektive Charakter der kollektiven kulturellen bzw. historischen Erinnerung und damit die Gültigkeit des Satzes: Keine Erinnerung ohne Vergessen. ${ }^{48}$ Gleichzeitig war damit ein Leidens- und Opfermythos geboren. Daraus ergab sich organisch der „Ruf nach Rache“ für die erlittene historische Niederlage auf dem Amselfeld. ${ }^{49}$ Dieser wurde wiederum zum Leitmotiv der nachfolgenden Kriege. Als konkrete Erinnerung gerann dieses zur unmittelbaren Motivation von Kampfestätigkeit, abzulesen etwa in den einschlägigen Quellen aus dem ersten serbischen Aufstand $1804-13^{50}$, den Kriegen im Verein mit Montenegro und in der Folge auch Russland gegen den osmanischen Oberherrn 1876-78 ${ }^{51}$, ganz besonders den Balkankriegen 1912/13 mit der Rückkehr des Kosovo nach Serbien ${ }^{52}$, in abgewandelter, generalisierter Form

Umbruchs- und Kriegszeiten, (volks-)literarisch positiv rezipiert, vgl. Beispiele bei Richter 1999, S. 386, 388, 389.

46 Vgl. hier auch Fußnote 20 dieses Aufsatzes.

47 Dazu Sundhaussen 2001, S. 24-27.

48 Vgl. die einleitenden Bemerkungen oben, insbesondere Fußnote 2.

49 Sundhaussen 2001, S. 26, 27.

50 Vgl. als Beispiel Matija Nenadovićs Beschreibung einer zentralen Rede des Aufstandsführers Karađorđe, in der dieser über die eigene Stellung und seine Aufgaben als Aufstandsführer sprach. Dabei trat als unmittelbares Motiv des Kampfes die Rache an den Türken für die von diesen getöteten Serben und für das „Joch, das der Serbe seit Kosovo bis heute trägt". Vgl. Stojančević 1985, S. 87.

51 Exemplarisch sei hier die Kriegsproklamation des serbischen Fürsten Milan Obrenović vom 1./13. Dezember 1877 genannt, in der es u.a. hieß: „Serben! Auch wir erheben uns nun zu den Waffen um der heiligen christlichen Sache unseres Volkes willen... Jetzt, nur noch ein entschlossener Schritt vorwärts und wir reichen uns die Hände mit jenen unseren Brüdern, von denen wir uns auf dem Kosovo verabschiedet haben..." Vgl. Ilić 1977, S. 33.

52 Vgl. dazu Höpken 2001, S. 378; wichtig hier auch die Berichte über die Mobilisierung des serbischen Heeres vor den Feldzügen ins Kosovo und Mazedonien vom Kriegskorrespondenten im Auftrag des Neusatzer Blattes Zastava Tomić 1999, S. 60. Kurz nach den Balkankriegen, am St.Veitstag 1914, schrieb der „Pijemont“, das einem militanten Nationalismus verpflichtete Organ der serbischen Geheimorganisation „Ujedinjenje ili smrt“, u.a.: „Diese heilige Flamme, die von Kosovo ausgehend Geschlechter begeisterte, ist zu einem mächtigen Feuer entflammt, von dem jeder Halm feindlicher Frech- 
auch in der serbischen Tschetnik-Bewegung im Zweiten Weltkrieg ${ }^{53}$, sowie nicht zuletzt in der schriftlichen und mündlichen Propaganda der nationalen Hochstimmung in den jüngsten jugoslawischen Zerfallskriegen. ${ }^{54}$ Erinnernd an die Amselfelder Schlacht schrieb der Belgrader Psychiater und SPSAbgeordnete im serbischen Parlament Jovan Striković in der Belgrader Politika unter bewusster Verwendung epischer Zehnsilber am 9. Mai 1992 u.a.: „Die Trompete vom Amselfeld seit diesem Tag jeden Tag in uns erklingt" ${ }^{55}$ Diese Aussage kann als - zum Handeln stimulierender - Fixpunkt einer auf lange Dauer angelegten Kosovo-Erinnerung gelten. Der Ethnologe Ivan Čolović hat dafür die folgenden treffenden Worte gefunden: „Die wichtigste Eigenart dieses kollektiven Erinnerns ist, dass es in einer ausnehmend intensiven Art lebendig zu sein vermag, dass es in einem extratemporalen Erleben mythischer Gegenwart die Gestaltung und Großtaten der heldenhaften Ahnen evozieren und sozusagen ,zurückholen' kann. Der Vidovdan ereignete sich ein einziges Mal, doch danach ereignet er sich - für den Serben - einmal im Jahr, am 28. Juni auf dem Gazimestan, oder sogar jeden Tag“. ${ }^{56}$ Deshalb müssen gerade heute die kursierende, schon seit Ende des 19. Jahrhunderts existierende Populärliteratur, aber auch die von Hand zu Hand wandernden Musik- und Videokasetten zum Thema ${ }^{57}$ in ihrer Bedeutung für das kollektive Erinnern besonders hervorgehoben werden. Gleichermaßen ist es wichtig, die offiziellen mobilisierenden Memorialhandlungen von Regierungen bzw. Herrschern einerseits, und den wissenschaftlichen und kulturellen Eliten zu den großen Jahres-

heit verbrannt wird. Durch eine Herculesleistung, die Jahrhunderte und Liebe vorbereiteten, werden Leid und Sklaverei beendet. Kosovo ist frei! Kosovo ist gerächt! Kosovos wird man ewig gedenken [...]; es wird ewig leben als Erinnerung aus der Vergangenheit und Beispiel für die Zukunft [... “. Im Übrigen kommt die nach der Eroberung des Kosovo durch serbische Truppen damals unter den Serben allgemein verbreitete nationale bzw. nationalistische Euphorie hier sehr gut zum Ausdruck. Zitat in Auszügen aus: Behschnitt 1980, S. 132.

53 „Die Tschetniks forderten [...] ein ethnisch ,gereinigtes Großserbien innerhalb Jugoslawiens und plädierten für die Umsiedlung und Vertreibung von fast drei Millionen Nicht-Serben (darunter Albaner). Ausdrücklich befürworteten sie das Prinzip der Rache für die an ihrer Nation begangenen Verbrechen: ,Sich nicht zu rächen, heißt so viel wie die Minderwertigkeit der eigenen Rasse anzuerkennen. Nur die kollektive und organisierte Rache wird die Wirkung der Rassenrache erzielen. Die Rache ist ein Problem der Ehre der serbischen Rasse““. Vgl. Sundhaussen 2001, S. 31; Zitat ebd. nach Tomasevich 1975, S. 166ff.

54 Vgl. Durić 1996, S. 9; Čolović 1994, S. 8.

55 Zitat nach Čolović 1994, S. 144.

56 Ebd. Das hier zutagetretende ganz spezielle Zeitverständnis (,gefrorene“ Zeit) wird im Folgenden näher erörtert.

57 Dazu Popović 2003, S. 51-57; 89-141; speziell für Montenegro Zirojević, S. 52 (Fußnote 7). Zur erwähnten „Populärliteratur“ seien exemplarisch hervorgehoben: Čajkanović 1918, sowie die in den Krisen und Kriegen im ehemaligen Jugoslawien zur nationalistischen Agitation benutzte Neuausgabe serbischer Heldenlieder: Đurić 1989. 
tagen der Amselfelder Schlacht andererseits (etwa 1888/1889 zum 500. oder 1989 zum 600. Jubiläum), in diesem Zusammenhang in den Blick zu nehmen. ${ }^{58}$ Beides zeugt von der besonderen Virulenz und Stärke der KosovoErinnerung als eines dominanten ,,mentalen Eintrags“ im kulturellen Gedächtnis der Serben und Montenegriner, bei beiden Völkern allerdings nicht in völlig gleicher Art und Weise.

Bei den Montenegrinern scheint nämlich die Erinnerung an die KosovoSchlacht von 1389 besonders tiefgehend verinnerlicht worden zu sein. Einen großen Anteil daran hat der oben schon genannte Dichterfürst Njegoš. Einerseits gelang ihm mit seinem berühmten, hier schon mehrfach erwähnten „Bergkranz" eine ethisch, religiös und national mobilisierend und erinnernd wirkende Allegorie auf das historische Ereignis auf dem Amselfeld. Dabei tritt das mythische Element der Rache des Miloš Obilić besonders hervor. Andererseits war auch seine Politik von einer lebendigen Erinnerung an die KosovoSchlacht durchwirkt. ${ }^{59}$ Er hielt den Obilić-Kult gezielt wach, verordnete der schwarz-roten Kopfbedeckung der Männer eine Veränderung und erweiterte Bedeutung: Die schwarze Seide sollte nun die Trauer um die verlorene Kosovo-Schlacht, der rote Stoff mit dem Wappen der Nemanjiden das Blut der serbischen Gefallenen symbolisieren. ${ }^{60}$ Wenige Jahrzehnte später beobachtete ein serbischer Zeitgenosse, dass die montenegrinischen Frauen auch bei ihren alltäglichen Verrichtungen ,,in Trauer nach dem Amselfeld“ seien und schwarze Kopftücher trügen. Wenn man mit ihnen spreche, habe man ,den Eindruck, dass die Amselfelder Schlacht gestern gewesen ist". Noch in der Zeit nach dem Zweiten Weltkrieg stiegen montenegrinische Hirtenmädchen auf den nächstgelegenen Berg, um dort gruppenweise die „Amselfelder Helden“ zu beweinen. ${ }^{61}$ Sie fühlten und fühlen sich z.T. heute noch als die Nachfahren jener kosovarischen Serben, die sich vor dem heranrückenden osmanischen Heer in die montenegrinischen Berge flüchteten. ${ }^{62}$ Somit wird die kollektive Erinnerung einer fernen Vergangenheit auch im ruhigen und sicheren Alltag zum unmittelbaren kollektiven Erleben der Gegenwart, die Vergangenheit erscheint gleichsam ,gefroren“, in einem ,liminalen“ Zeitverständnis, wie es Klaus Roth ausgedrückte. ${ }^{63}$ In diesem Extrem könnte man einen eher graduel-

58 Zum 500. Gedenktag vgl. Höpken 2001, S. 378, hier mit ausführlichen Belegen (in Fußnote 33); Nitsche 2003, S. 147; zum 600. Gedenktag mit seinen Massenveranstaltungen Zirojević 1999, S. 59, 60, ferner Sundhaussen 2001, S. 33, 34.

59 Miedlig 2006, S. 157.

60 Popović 2003, S. 94.

61 Zirojević 1999, S. 52, insbes. hier Fußnote 6. Was die ersten beiden Belege, die die 2. Hälfte des 19. Jahrhunderts betreffen, anlangt, so folgt die Autorin dem Zeugnis Ljubomir Nenadovićs. Vgl. dazu Nenadović 1929, S. 20.

62 Popović 2003, S. 94.

63 Vgl. Roth 1995, S. 33, 36-38. Der Soziologe Daniel Šuber beschreibt dieses Phänomen, ausgehend von einem kulturalistisch-hermeneutischen Ansatz, in Anlehnung an die Forschungen des amerikanischen Kultursoziologen Jeffrey C. Alexander wie folgt: 
len, keineswegs grundsätzlichen Unterschied zur entsprechenden Situation bei den Morava-Serben sehen, wo dieses Zeitverständnis besonders seit der 2 . Hälfte des 19. Jahrhunderts vor allem in Kriegen und staatlichen bzw. gesellschaftlichen Umbruchskrisen sichtbar und auch historisch fassbar wird.

Weitere Detailforschungen zu den angesprochenen Themen sind geboten, denn die Erforschung der südosteuropäischen Erinnerungskulturen im allgemeinen und die der Serben und Montenegriner im Besonderen stecken nach wie vor in den Kinderschuhen. Sie werden über den hier gegebenen Überblick hinaus - etwa durch geographisch engräumige und historisch „dichte“ local studies - vertiefte (Er-)Kenntnisse zutagefördern.

\section{Literaturverzeichnis}

Alimpić, Mileva, 1852: Život i rad generala Ranka Alimpića. Napisala njegova udovica Mileva. Beograd.

Behschnitt, Wolf Dietrich, 1980: Nationalismus bei Serben und Kroaten 1830 - 1914. Analyse und Typologie der nationalen Ideologie (= Südosteuropäische Arbeiten; 74). München.

Braun, Maximilian (Hrsg.), 1956: Die Lebensbeschreibung des Despoten Stefan Lazarević. Im Auszug herausgegeben und übersetzt von Maximilian Braun (= SlavoOrientalia; 1). Wiesbaden.

Braun, Maximilian, 1961: Das serbokroatische Heldenlied (= Opera Slavica; 1). Göttingen.

Butzer, Günter / Günter, Manuela (Hrsg.), 2004: Kulturelles Vergessen. Medien - Rituale - Orte, (= Formen der Erinnerung; 21). Göttingen.

Čajkanović, Veselin, 1918: „Iz srpske religije i mitologije. 1. Majka Jugovića“, in: Zabavnik. Dodatak Srpskih novina, 15. decembar 1918.

Čolović, Ivan, 1994: Bordell der Krieger. Folklore, Politik und Krieg. Osnabrück.

Dašić, Miomir, 1983: „Uticaj Prvog srpskog ustanka na nacionalno-oslobodilački pokret u Vasojevićima i ostalim crnogorskim brdima (1804-1813)“, in: Čubrilović, Vasa (Hrsg.): Istorijski značaj srpske revolucije 1804. godine. Zbornik radova sa naučnog skupa od 3. do 5. juna 1980, povodom obeležavanja 175. godišnjice Prvog srpskog ustanka (= SANU. Naučni skupovi, knj. XVIII. Odeljenje istorijskih nauka, knj. 6). Beograd, 545-598.

Durić, Rašid, 1996: „Der nationale Mythos in der serbischen Literatur und Politik“, in: Österreichische Osthefte 38 (1996), H. 1, 3-21.

„Der Effekt einer Präsentation von vorgeblich realen Verhältnissen entsprechend dem mythischen Skript des Deutungsmusters [des Kosovo-Mythos - Erg. von H.-M. Miedlig] lässt sich in einer Verschmelzung (mythischer) Vergangenheit und aktueller Gegenwart zusammenfassen. Performanztheoretiker bezeichnen diese Auflösung der Grenze zwischen Vergangenheit und Gegenwart beziehungsweise Skript und Performanz als „Fusion“. Vgl. Suber, 2004, S. 371. Damit gelangt er in seiner Disziplin letztendlich zum gleichen Ergebnis wie der Volkskundler Roth. 
Đurić, Vojislav, 1989: Antologija narodnih junačkih pesama. 12. izdanja. Beograd.

Emmert, Thomas A., 1990: Serbian Golgatha. Kosovo 1389 (= East European Monographs; 278). New York.

Erll, Astrid, 2005: Kollektives Gedächtnis und Erinnerungskulturen. Eine Einführung. Stuttgart, Weimar.

Fromm, Martin / Haase, Frank / Schlottke, Peter F., 2003: „Vorwort der Herausgeber", in: Fromm, Martin / Haase, Frank / Schlottke, Peter F. (Hrsg.): Erfindung der Vergangenheit. München, 7.

Hoepken, Wolfgang, 1999: „War, Memory, and Education in a Fragmented Society: The Case of Yugoslavia“, in: East European Politics and Societies 13 (1999), No. 1, 190-227.

Höpken, Wolfgang, 2001: „Kriegserinnerung und Kriegsverarbeitung auf dem Balkan. Zum kulturellen Umgang mit Kriegserfahrungen in Südosteuropa im 19. und 20. Jahrhundert“, in: Südosteuropa-Mitteilungen 41 (2001), H. 4, 371-389.

Ilić, Nikola P., 1977: Oslobođenje južne Srbije 1877-1878. Beograd.

Jaworski, Rudolf, 2003: „Alte und neue Gedächtnisorte in Osteuropa nach dem Sturz des Kommunismus“, in: Jaworski, Rudolf / Kusber, Jan / Steindorff, Ludwig (Hrsg.): Gedächtnisorte in Osteuropa: Vergangenheiten auf dem Prüfstand. Frankfurt, Berlin u.a., 11-25.

Kilibarda, Novak, 1983: „Vožd Karađorđe u pjesničkoj projekciji Njegoša i F. Višnjića“, in: Čubrilović, Vasa (Hrsg.): Istorijski značaj srpske revolucije 1804. godine. Zbornik radova sa naučnog skupa od 3. do 5. juna 1980, povodom obeležavanja 175. godišnjice Prvog srpskog ustanka (= SANU. Naučni skupovi, knj. XVIII. Odeljenje istorijskih nauka, knj. 6). Beograd, 787-794.

Ljušić, Radoš, 1994: „Višnjić, Vuk i boj na Loznici“, in: Ders.: Srbija 19. veka. Izabrani radovi. Beograd, 380-382.

Miedlig, Hans-Michael, 1991: „Patriarchalische Mentalität als Hindernis für die staatliche und gesellschaftliche Modernisierung in Serbien im 19. Jahrhundert", in: Südost-Forschungen 50 (1991), 163-190.

Miedlig, Hans-Michael, 2006: „Zur Frage der Ethnizität und Identität der Montenegriner in Geschichte und Gegenwart", in: Zeitschrift für Balkanologie 42 (2006), 1+2, 146-165.

Miljković, Branislav, 1977: „Đaci i studenti do prvog svetskog rata“, in: Savić, Pavle et al. (Hrsg.): Beograd u sé́anjima 1900-1918. Beograd, 71-85.

Nenadović, Ljubomir, 1929: O Crnogorcima. Pisma iz Cetinja 1878. godine (= Srpska Književna Zadruga; 212). Beograd.

Nikolić, Milorad T., 1983: „Prvi i drugi ustanak na sceni Narodnog Požorišta u Beogradu“, in: Čubrilović, Vasa (Hrsg.): Istorijski značaj srpske revolucije 1804. godine. Zbornik radova sa naučnog skupa od 3. do 5. juna 1980, povodom obeležavanja 175. godišnjice Prvog srpskog ustanka (= SANU. Naučni skupovi, knj. XVIII. Odeljenje istorijskih nauka, knj. 6). Beograd, 813-830.

Nitsche, Peter, 2003: „,Das serbische Golgatha‘. Das Kosovo als Gedächtnisort und nationaler Mythos", in: Jaworski, Rudolf / Kusber, Jan / Steindorff, Ludwig (Hrsg.): Gedächtnisorte in Osteuropa: Vergangenheiten auf dem Prüfstand. Frankfurt, Berlin u.a., 139-156. 
Oexle, Otto Gerhard, 1995: „Memoria als Kultur“, in ders. (Hrsg.): Memoria als Kultur. Göttingen, 9-78.

Olshausen, Eckart, 2003: „Der Umgang mit der Vergangenheit in den homerischen Gesellschaften“, in: Fromm, Martin / Haase, Frank / Schlottke, Peter F. (Hrsg.): Erfindung der Vergangenheit. München, 159-166.

Petrović, Dušan, 1976: „Osnovni ciljevi prvog srpskog ustanka“, in: Istorijski časopis XXIII (1976), 51-60.

Popović, Sonja, 2003: Die Mythologisierung des Alltags. Kollektive Erinnerungen, Geschichtsbilder und Vergangenheitskultur in Serbien und Montenegro seit Mitte der 1980er Jahre (= Basler Studien zur Kulturgeschichte Osteuropas; 5). Zürich.

Possart, Paul Anton Fedor Konstantin (Prof. Dr. P.A. Fed. Konst. Possart - Mitbegründer des Vereins für gemeinnützige, insbesondere vergleichende Kunde der Hauptsprachen Europas, in Frankfurt a.M.), 1837: Das Fürstenthum Serbien, seine Bewohner, deren Sitten und Gebräuche. I. Abteilung. Historisches und Geographisches. Darmstadt.

Richter, Angela, 1999: „Rückgriffe auf den Vidovdan-Mythos in literarischen Werken des 20. Jahrhunderts“, in: Behring, Eva / Richter, Ludwig / Schwarz, Wolfgang (Hrsg.): Geschichtliche Mythen in den Literaturen und Kulturen Ostmittel- und Südosteuropas (= Forschungen zur Geschichte und Kultur des östlichen Mitteleuropa; 6). Stuttgart, 381-392.

Roth, Klaus, 1995: „Zeit, Geschichtlichkeit und Volkskultur im postsozialistischen Südosteuropa“, in: Zeitschrift für Balkanologie 31 (1995), H. 1, 31-45.

Schubert, Gabriella, 1996: „Auf den Spuren von Königssohn Marko“, in: Hardten, Eggert u.a. (Hrsg.): Der Balkan in Europa. Frankfurt am Main, Berlin u.a., 151-164.

Stefanović, Dragutin A. / Stanisavljević, Vukašin, 1968: Pregled jugoslovenske književnosti. Knj. I: Narodna književnost, feodalna književnost, dubrovačko-dalmatinska književnost i racionalizam. Beograd.

Steindorff, Ludwig, 2003: „Schichten der Erinnerung. Zur Klassifizierung von Gedächtnisorten in Kroatien“, in: Jaworski, Rudolf / Kusber, Jan / Steindorff, Ludwig (Hrsg.): Gedächtnisorte in Osteuropa. Vergangenheiten auf dem Prüfstand (= Kieler Werkstücke. Reihe F: Beiträge zur osteuropäischen Geschichte; 6). Frankfurt, Berlin u.a., 157-182.

100 najznamenitijih Srba. Beograd, Novi Sad 1993.

Stojančević, Vladimir, 1985: „Srbija, zemlja i ljudi, u delu prote Matije za vreme prvog srpskog ustanka“, in: Samardžić, Radovan (Hrsg.): Prota Matija Nenadović i njegovo doba. Zbornik radova sa naučnog skupa održanog od 5. do 7. decembra 1978. godine (= SANU: Naučni skupovi, knj. XXVI. Predsedništvo, knj. 6). Beograd, 83-95.

Šuber, Daniel, 2004: „Kollektive Erinnerung und nationale Identität in Serbien. Zu einer kulturalistischen Interpretation des Anfangs vom Ende Jugoslawiens", in: Giessen, Bernhard / Schneider, Christoph (Hrsg.): Tätertrauma. Nationale Erinnerungen im öffentlichen Diskurs (= Historische Kulturwissenschaft; Band 2). Konstanz, 347-379.

Sundhaussen, Holm, 1993: Experiment Jugoslawien. Von der Staatsgründung bis zum Staatszerfall. Mannheim u.a. 
Sundhaussen, Holm, 2001: „Kriegserinnerung als Gesamtkunstwerk und Tatmotiv: Sechshundertzehn Jahre Kosovo-Krieg (1389-1999)“, in: Beyrau, Dietrich (Hrsg.): Der Krieg in religiösen und nationalen Deutungen der Neuzeit. Tübingen, 11-40.

Tomasevich, Jozo, 1975: War and Revolution in Yugoslavia, 1941-1945. The Chetniks. Stanford/Cal.

Tomić, Jaša, 1999: Rat na Kosovu i Staroj Srbiji 1912. godine. Drugo fototipsko izdanje [prema izd. iz 1913]. Niš.

Veselinović, Rajko L., 1976: „Razvitak misli o srpskoj državnosti u oslobodilačkim pokretima i ustancima tokom XVI i XVII stoleća“, in: Simpozijum: Oslobodilački pokreti jugoslovenskih naroda od XVI veka do početka prvog svetskog rata (= Istorijski Institut. Zbornik radova; 1). Beograd, 33-56.

Žanić, Ivo, 1998: Prevarena povijest. Guslarska estrada, kult hajduka $i$ rat u Hrvatskoj $i$ Bosni i Hercegovini 1990-1995. godine. Zagreb.

Zirojević, Olga, 1999: „Das Amselfeld im kollektiven Gedächtnis“, in: Melčić, Dunja (Hrsg.): Der Jugoslawienkrieg. Handbuch zu Vorgeschichte, Verlauf und Konsequenzen. Opladen, Wiesbaden, 45-61.

Živanović, Žarko D. (Prof. Dr. Žarko D. Živanović), 1991: „Potvrda nacionalnog identiteta", in: Politika, 15. januar. 
